\title{
Avaliação da Dinâmica da Vegetação em Áreas Desmatadas na Floresta Amazônica
}

\author{
Roberto Diego Bezerra dos Santos ${ }^{1}$, Rafael Coll Delgado ${ }^{1}$, \\ Emanuel José Gomes de Araújo², Eduardo Vinícius da Silva²
}

${ }^{1}$ Departamento de Ciências Ambientais, Universidade Federal Rural do Rio de Janeiro - UFRRJ, Seropédica/RJ, Brasil ${ }^{2}$ Departamento de Silvicultura, Instituto de Florestas, Universidade Federal Rural do Rio de Janeiro - UFRRJ, Seropédica/RJ, Brasil

\begin{abstract}
RESUMO
No presente estudo avaliou-se a dinâmica da vegetação de florestas tropicais no período de 2000 a 2010 em áreas desmatadas, utilizando-se os produtos MOD44B, 3B43 e SRTM. Através deles analisou-se, temporal e espacialmente, a ocorrência de regeneração em áreas de desmatamento mapeadas pelo sistema PRODES (Projeto de Estimativa de Desflorestamento da Amazônia) do INPE (Instituto Nacional de Pesquisas Espaciais). Os resultados encontrados mostraram que há regeneração nas áreas desmatadas e que, ao longo do tempo, a densidade da vegetação tende a aumentar. As áreas regeneradas apareceram majoritariamente em áreas mais baixas, seguindo a tendência do desmatamento. O MOD44B mostrou-se eficiente no estudo temporal e espacial da vegetação, indicando haver regeneração ao longo do tempo. O período de maior precipitação coincidiu com o período em que os pixels de menor densidade migraram para classes de maior densidade, sugerindo uma possível relação entre os índices de precipitação e o crescimento da vegetação.
\end{abstract}

Palavras-chave: crescimento da vegetação, análise estatística, sensoriamento remoto, MODIS.

\section{Vegetation Dynamics Evaluation in the Amazon Rainforest Deforested Areas}

\begin{abstract}
This article uses data from orbital platforms (MOD44B, 3B43 and SRTM) to evaluate the vegetation dynamics of tropical forests in deforested areas between 2000 and 2010. We analyzed, temporally and spatially, the occurrence of vegetation regeneration in deforested areas mapped by the PRODES system (Programa de Cálculo do Desflorestamento da Amazônia) of the INPE (Instituto Nacional de Pesquisas Espaciais). The results revealed regeneration in deforested areas and also showed that vegetation density tends to increase over time. Areas of regeneration are mainly found in low land areas following the path of deforestation. MOD44B was effective in the vegetation temporal and spatial study, and indicated occurrence of regeneration over time. The higher precipitation period overlapped with the period in which lower density pixels migrated to classes of higher density, which suggests a possible relationship between precipitation indexes and vegetation growth.
\end{abstract}

Keywords: vegetation growth, statistical analysis, remote sensing, MODIS. 


\section{INTRODUÇÃO}

Compreender a dinâmica de desflorestamento e regeneração natural em florestas tropicais é vital para a conservação e manejo desses ecossistemas. Sua localização geográfica, elevada disponibilidade de biomassa e de água fazem com que essas áreas sejam alvo de constantes intervenções humanas.

A Amazônia brasileira vem, há décadas, sofrendo com a intensa degradação de sua vegetação natural devido, principalmente, à criação de rodovias, desenvolvimento de polos no processo de ocupação (Machado, 1998; Alves et al., 1999, 2009; Alves, 2002) e à intensificação do uso da terra e expansão de novas áreas desmatadas, associadas ao abandono após o esgotamento dos recursos delas.

Outro fator de destaque, atualmente, é a criação de usinas hidrelétricas que, além de contribuírem com a perda de cobertura vegetal, também contribuem com a emissão de gases de efeito estufa, como o metano (Fearnside, 2012).

Quantificar as variações da cobertura florestal amazônica e compreender as variáveis que as influenciam em escala regional ou continental é importante, devido a seu papel na fixação do carbono global (Malhi \& Grace, 2000) e na contribuição no ciclo hídrico da região (Mcguffie et al., 1995; Werth \& Avissar, 2002).

Entretanto, estudos de vegetação com grande abrangência territorial nessa região por meio de amostragens de campo representativas são difíceis de serem realizados, principalmente devido à sua extensa área, heterogeneidade, hostilidade, relevo e dificuldade de deslocamento (Aragão et al., 2007).

Nessas situações, o uso de dados obtidos via sensoriamento remoto permite estudos de alta confiabilidade nas escalas espaciais e temporais na região amazônica, recobrindo periodicamente diversas regiões do planeta, sendo esses dados reajustados continuamente (Freitas et al., 2012; Vilanova et al., 2013).

Um dos sensores mais usados em estudos em grandes extensões territorais é o MODIS (Moderate Resolution Imaging Spectroradiometer). Seus dados estão disponíveis desde 2000, constituindo assim uma das fontes mais confiáveis no que se refere a estudos de pequena escala ou até mesmo de escala global (Zhang et al., 2009).
Recentemente, novos sensores de resolução média com cobertura da Amazônia brasileira estão se tornando ferramentas eficientes na avaliação do desmatamento. O MODIS é um dos mais completos registros no que se refere à identificação de áreas florestadas, sendo responsável por gerar o produto MOD44B, também denominado Vegetation Continuous Field (VCF). O MODIS é um dos cinco sensores a bordo do satélite TERRA e AQUA da NASA EOS (Earth Observing System - Sistema de Observação da Terra) (Townshend et al., 2001). Esse sensor oferece imagens com resolução espacial de $250 \mathrm{~m}, 500 \mathrm{~m}$ e $1.000 \mathrm{~m}$, possui 36 bandas espectrais e cobre o espectro eletromagnético da região do visível ao infravermelho termal (Running et al., 1994).

Outro produto de sensoriamento remoto é o satélite TRMM (Tropical Rainfall Measuring Mission), e seu produto, 3B43 (Huffman et al., 2007), fornece informações horárias de precipitação para todas as regiões tropicais do planeta, suprindo a defasagem causada pela ausência de estações meteorológicas em certas regiões.

Em relação aos dados de radares, outra geotecnologia, consiste na utilização de dados de relevo, gerados a partir de dados de radar, obtidos de sensores a bordo do ônibus espacial Endeavour, no projeto SRTM (Shuttle Radar Topography Mission). O Modelo Digital de Elevação (MDE) do SRTM, com $3 \mathrm{~s}$ de arco (aproximadamente 90 m de resolução espacial), é distribuído gratuitamente pelo governo norte-americano (Miranda, 2005).

O objetivo foi aplicar o produto MOD44B para identificação e avaliação do desenvolvimento da regeneração natural em áreas desmatadas da floresta amazônica mapedas pelo INPE no período de 2000 a 2010, utilizando o produto MOD44B, e avaliar a relação da regeneração natural com a variável precipitação e relevo, através dos produtos 3B43 e SRTM

\section{MATERIAL E MÉTODOS}

\subsection{Caracterização da área de estudo}

Situado na Região Norte do Brasil, o município de Plácido de Castro, AC, possui área superficial de $1.943,245 \mathrm{~km}^{2}$ (IBGE, 2013) (Figura 1). O clima predominante é Aw, segundo classificação climática de Köppen. O estado do Acre é dividido em dois 


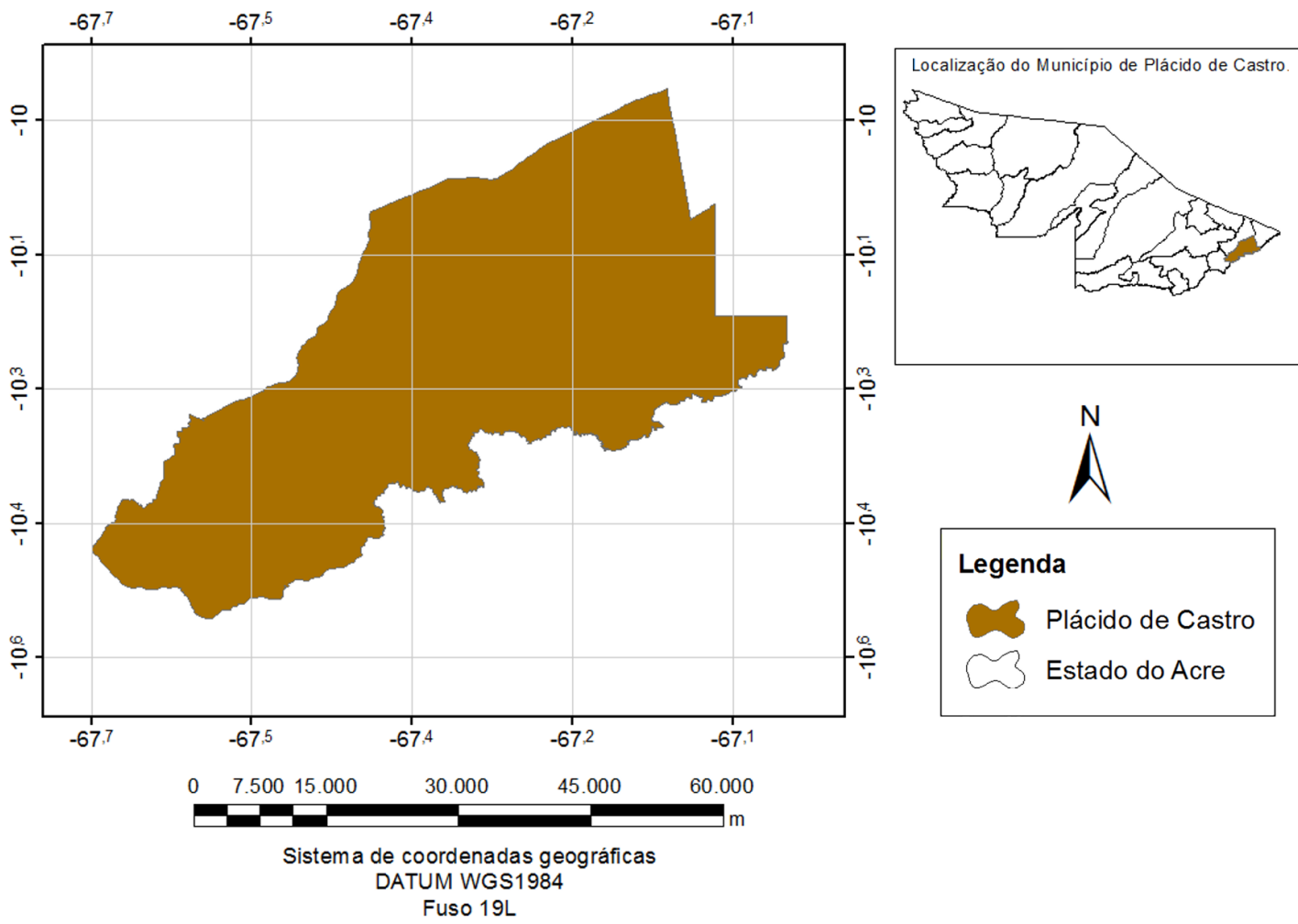

Figura 1. Localização geográfica da área de estudo e o uso da terra gerado pelo produto MCD12Q1 para o município de Plácido de Castro, AC.

Figure 1. Geographic location of the study area and the land use generated by MCD12Q1 product to the municipality of Plácido de Castro, AC.

principais tipos de clima, sendo eles: tropical úmido (Af) e tropical de monções (Am) (Duarte, 2005).

\subsection{Obtenção e processamento dos dados}

Neste estudo foram utilizadas 11 imagens, compreendendo os anos de 2000 a 2010, do produto MOD44B, oriundo do sensor MODIS, a bordo da plataforma orbital TERRA, com composição anual formada pelas imagens dos 12 meses que resultam na imagem final e resolução espacial de $250 \mathrm{~m}$. As imagens foram adquiridas no dia 22 de abril de 2013 da NASA (National Aeronautics and Space Administration) (USGS, 2013). Utilizou-se também o polígono referente ao desmatamento mapeado pelo PRODES (Projeto de Estimativa de Desflorestamento da Amazônia) do ano 2000, desenvolvido pelo INPE - Instituto Nacional de Pesquisas Espaciais (INPE, 2011).

Os dados MOD44B foram utilizados para a realização da leitura dos pixels da densidade da cobertura de vegetação nos polígonos PRODES do 2000, para o acompanhamento da dinâmica de regeneração ao longo de 11 anos, no município de Plácido de Castro, AC.

As imagens foram disponibilizadas em formato HDF (Hierarchical Data Format) e projeção Sinusoidal. Para que as imagens pudessem ser processadas no ArcGis 10.1, foi necessária transformação do formato HDF para GEOTIFF e da projeção sinusoidal para UTM WGS 84, zona 19L. Para isso, realizou-se um pré-processamento das imagens no algoritmo do MRT (Modis Reprojection Tool). O MRT é um software exclusivo para trabalhar com imagens MODIS (USGS, 2013).

Utilizou-se o mapa de uso e ocupação do solo, produto MCD12Q1, disponibilizado pelo EOSDIS - NASA's Earth Observing System Data and Information System (NASA EOSDIS, 2013), para realizar a separação de áreas de regeneração espontânea, identificadas através do MOD44B, dentro de polígonos de desmatamento do sistema PRODES.

Outro procedimento consistiu na utilização de dados de relevo, gerados a partir de dados de radar, 
obtidos dos sensores a bordo do ônibus espacial Endeavour, no projeto SRTM (Shuttle Radar Topography Mission). Em seguida, com o SRTM, geraram-se os mapas de elevação, depois as informações obtidas pelo MOD44B foram sobrepostas ao mapa de elevação, a fim de verificar em quais altitudes estariam as áreas em regeneração. Todos esses procedimentos foram realizados no software ArcGIS 10.1.

Para a avaliação do comportamento da precipitação, utilizaram-se imagens TRMM (Tropical Rainfall Measuring Mission), com resolução espacial de $15 \mathrm{~km}$ (TRMM, 2013). Os valores de precipitação de cada pixel do TRMM foram extraídos para o município e seu entorno e, posteriormente, obteve-se a média para cada ano. Em seguida, avaliou-se a possível relação do avanço da regeneração natural com o comportamento da precipitação.

O produto 3B43 foi disponibilizado no formato raster.nc (NetCDF), sendo utilizado o programa ArcGIS 10.1 para a conversão e leitura em planilhas. No software ArcGIS 10.1, foram utilizados o ArcToolbox - Multidimension Tools e as ferramentas de conversão Make NetCDF Raster Layer e Make NetCDF Table View.

Após o pré-processamento das imagens, no software ArcGIS 10.1, módulo Arctoolbox - Analysis Tools Extract - e através da ferramenta clip, elaborou-se o recorte do município de Plácido de Castro do polígono PRODES, no estado do Acre. Posteriormente, adicionouse a imagem do produto MOD44B. Em seguida foi elaborado o recorte da imagem MOD44B, com base nos polígonos do PRODES situados em Plácido de Castro.

As imagens raster do MOD44B foram transformadas em pontos para facilitar a contagem dos índices em escala percentual de cobertura da vegetação encontrada em cada polígono. Para isso utilizou-se o módulo Arctoolbox - Convertion Tools - From Raster - Raster to Point (Figura 2).

Os polígonos PRODES foram classificados em três grupos: "desflorestamento referente ao ano da imagem", "floresta" e "desmatamento". Selecionaram-se os dados referentes à classificação encontrada na tabela de atributos PRODES. Assim, com a ferramenta Arctoollbox - analist tools - extract - clip foram elaborados os mapas com os pontos de cada grupo. Para a classificação do grupo "floresta", selecionaram-se todos os polígonos referentes aos anos posteriores ao ano da imagem. O grupo "desmatamento" referiu-se aos polígonos que foram posteriormente adicionados ao PRODES em 2010. Repetiu-se esse procedimento em todas as imagens MODIS do período de estudo.

Os dados da tabela de atributos nos mapas de cada grupo nas imagens MOD44B de 2000 a 2010 foram exportados do ArcGis 10.1 para o software Excel 2013. Nos dados exportados reuniram-se as informações sobre todos os valores dos pixels do MOD44B encontrados no interior de cada polígono do PRODES.

De acordo com a metodologia proposta por Vilanova et al. (2013), os índices de porcentagem

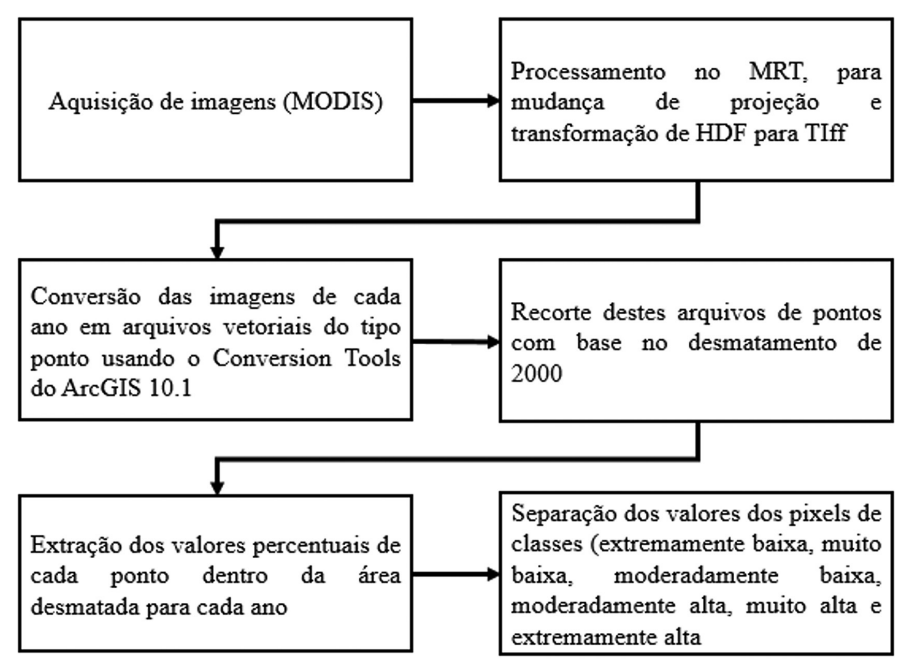

Figura 2. Fluxograma referente ao processamento dos dados do MODIS.

Figure 2. Flowchart related to MODIS data processing. 
de cobertura florestal foram divididos em categorias consideradas como: Extremamente baixo (0\% a 5\% - EB), Muito baixo (5\% a $17 \%$ - MB), Moderadamente baixo (17\% a $35 \%$ - MODB), Moderadamente alto (35\% a $54 \%$ - MODA), Muito alto (54\% a $71 \%$ - MA) e Extremamente alto (71\% a 89\% - EA), adotando-se a simbologia das classes (EB, MB, MODB, MODA, MA e EA). Vale ressaltar que os pixels das imagens MOD44B analisados não apresentaram valores maiores que $89 \%$ por cento, por essa razão não há classe percentual acima desse valor.

Para essa seleção, adotou-se o Box plot exploratório, a partir dos valores de cada pixel das imagens de 2000 a 2010, selecionou-se cada categoria individualmente para cada ano de acordo com a ordem percentual correspondente ao valor de cada pixel das imagens.

\subsection{Análise estatística}

Foi feita a análise descritiva da variável índice de porcentagem da cobertura florestal dos pixels, onde se estimou a média, a variância, o desvio-padrão e o coeficiente de variação para cada ano em cada categoria considerada para as imagens MOD44B.

Para compreender a evolução de cada categoria ao longo do tempo, aplicou-se análise de regressão, utilizando-se o modelo linear múltiplo do segundo grau com o número de pixels da respectiva classe como variável dependente em função da variável independente tempo. Isso permitiu compreender se, ao longo do tempo, o número de pixels de uma classe tende a reduzir ou aumentar, caracterizando desflorestamento ou crescimento. Possíveis diferenças entre as frequências de pixels nas categorias de cobertura florestal foram analisadas por meio do teste de Kolmogorov-Smirnov, com 5\% de significância, conforme descrito em Araújo et al. (2012). Esse teste é muito aplicado para comparar a frequência diamétrica entre florestas (Scolforo \& Thiersch, 2006), o qual neste trabalho foi adotado para comparar a frequência de pixels. Isso foi feito para verificar se os pixels presentes nas categorias com pouca vegetação estavam migrando para as categorias com vegetação mais densa ao longo do tempo. Como florestas com alta diversidade possuem uma taxa de crescimento lenta, é necessário analisar o incremento de vegetação entre um período de tempo de no mínimo três anos.
Assim, foram selecionadas as categorias de cobertura florestal nos anos 2000, 2003, 2006 e 2010.

Para a análise dos dados de precipitação foram extraídos os valores de cada pixel das imagens do produto 3B43, para cada mês, de cada ano do período estudado. A partir desses valores foram calculadas as médias anuais e buscou-se verificar a possível ocorrência de anos secos e chuvosos e se esses períodos coincidiam com os momentos de crescimento das densidades dos pixels de vegetação florestal em regeneração.

\section{RESULTADOS E DISCUSSÃO}

A Figura 3 mostra o comportamento de cada classe de densidade da cobertura florestal, no período de 2000 a 2010, na área desmatada, referente ao ano de 2000. No primeiro gráfico (Figura 3a), observa-se o comportamento da classe EB, a qual apresenta um padrão inicial de ascensão e depois de decréscimo ao longo do período estudado.

A Figura 3 b refere-se à ocorrência dos pixels dentro da classe MB nos 11 anos analisados. De 2000 a 2006, o número de pixels aumenta, entretanto, a partir de 2006 a curva mostra um comportamento descendente, indicando que os pixels existentes nessa classe podem estar sofrendo mudança em seus valores percentuais e migrando para outras classes com o passar dos anos. O coeficiente de determinação da Figura $3 b\left(r^{2}=0,5363\right)$ indica que $53,63 \%$ da variação dos pixels tem relação com o passar dos anos.

A Figura $3 c$ apresenta o coeficiente de determinação $r^{2}=0,5003$. A variação da curva de distribuição dos pixels para a classe MODB mostra um discreto crescimento do número de pixels até o ano 2006. A partir desse ano, a curva se acentua ainda mais no sentido ascendente, indicando o crescimento do número de pixels na classe. Já a classe MODA (Figura 3d), com r ${ }^{2}=0,8452$, mostra acentuado decréscimo do número de pixels. Porém, a partir do ano 2006, a curva assume comportamento ascendente. $\mathrm{O}$ mesmo ocorre na Figura 3e, com $r^{2}=0,6611$. Já a Figura $3 f$ apresenta comportamento descendente até o fim do período estudado. Entretanto, essa classe representa uma vegetação num estágio de desenvolvimento mais avançado, sugerindo que, numa análise dos anos subsequentes ao ano 2010, a curva possa apresentar um comportamento ascendente. 

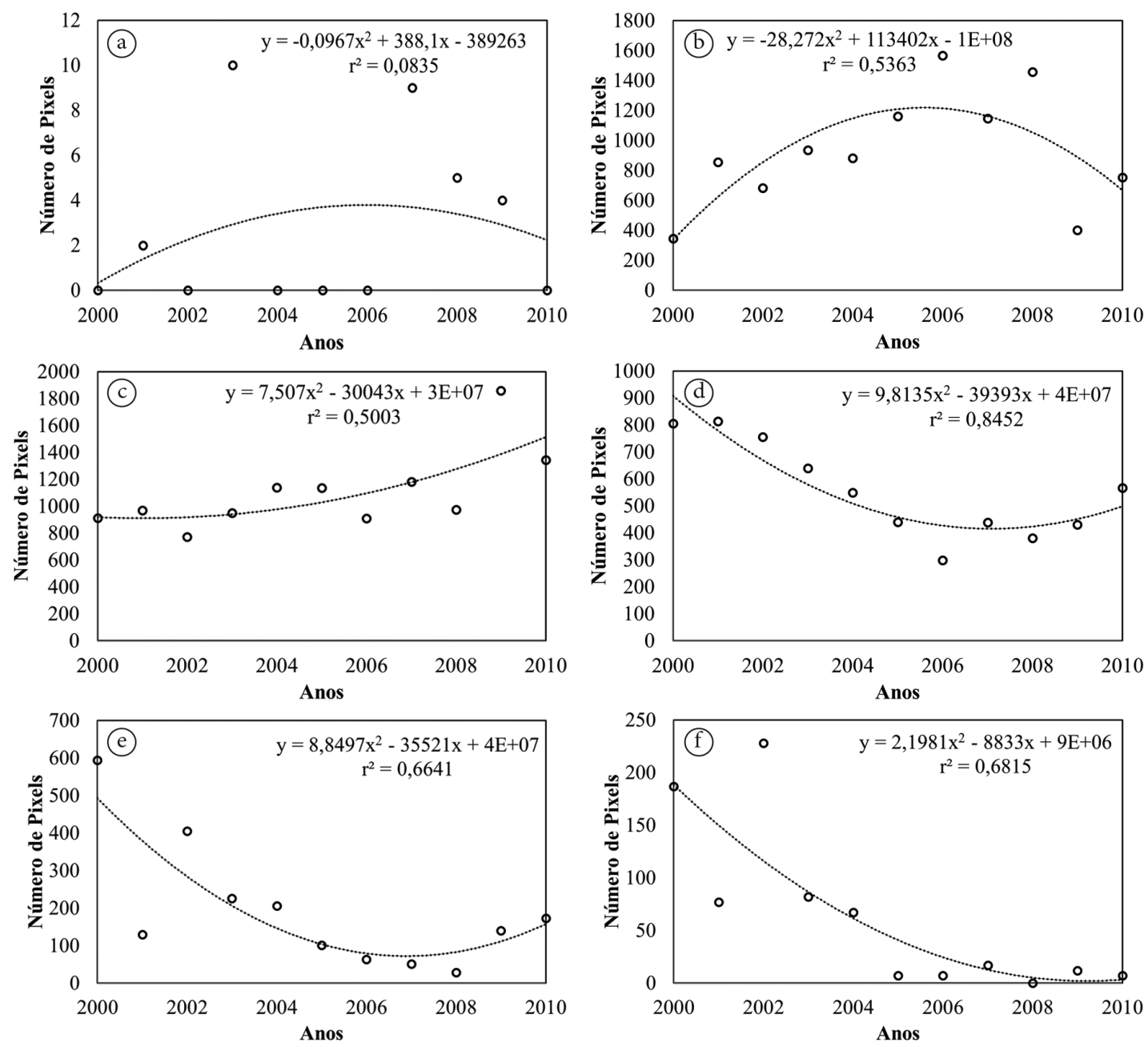

Figura 3. Análise de regressão das categorias EB (a), MB (b), MODB (c), MODA (d), MA (e) e EA (f) para os anos de 2000 a 2010

Figure 3. Regression analysis of the EB categories (a), MB (b), MODB (c) MODA (d) and EA (e) for the years 2000-2010.

De maneira geral, excetuando-se a curva EA, o aumento nas curvas de maior densidade coincide com o momento de declínio da classe MB, no caso, o ano 2006. Esse comportamento das curvas evidencia a variação das características estruturais da vegetação na qual, por exemplo, a cobertura vegetal predominantemente composta por arbustos e gramíneas passa a dar lugar a indivíduos arbóreos mais adensados e com maior cobertura de copa.

Dados do mapeamento de desmatamento na Amazônia no período de 2000 a 2010 (IMAZON, 2013) e do PRODES mostram que a partir de 2005 ocorre redução significativa nas taxas de desmatamento no estado do Acre, permanecendo em queda até o fim do período estudado. Uma área desmatada que se regenerou pode, futuramente, ser desmatada novamente, entretanto, segundo o referido mapeamento, o estado do Acre vem controlando esses processos de desmatamento, resultando num declínio dessa atividade.

Segundo Soares-Filho et al. (2005), a pavimentação e a construção de estradas consistem o principal determinante dos padrões futuros de desmatamento da região Amazônica. O mesmo não ocorre no Acre nesta análise. O que pode estar contribuindo para o declínio do desmatamento observado por sensoriamento remoto são as florestas plantadas de Teca (Tectona grandis L. f.), no sul do Acre (Vilanova et al., 2013). 
A avaliação estatística do comportamento dos valores dos pixels dentro das categorias (desvio-padrão, média e coeficiente de variação) está representada na Tabela 1a, b. O comportamento das médias dentro das categorias ao longo do tempo se manteve praticamente constante. Entretanto, ao longo dos anos, a quantidade de pixels diminui dentro das classes de menor densidade. Indicando que os pixels que permanecem dentro delas são os pixels com os maiores valores dentro dos intervalos, indicando que esses, futuramente, migrarão para classes de maior densidade.

A categoria que obteve os maiores valores médios foi a $E A$, com valores entre $73,57 \%$ e $75,89 \%$, e a que apresentou os maiores valores foi a $\mathrm{MB}$, com valores entre $10,96 \%$ e $14,38 \%$.

O desvio-padrão para cada categoria apresentou baixa variabilidade. A categoria que apresentou o maior desvio-padrão foi a MODA, com valores entre $5,29 \%$ e $5,93 \%$, e a que apresentou os menores valores foi a categoria $\mathrm{MB}$, com valores entre 1,54\% e 2,43\%. De forma geral o desvio-padrão de cada classe diminuiu durante o período estudado, exceto na classe EA.

Já o coeficiente de variação $(\mathrm{CV})$ diminuiu das classes de menor porcentagem para as de maior porcentagem. E, dentro de cada categoria, o CV diminuiu ao longo do tempo. A categoria que apresentou maior variação do CV foi a MB, com valores entre 10,68\% e 19,15\%, indicando grande variação em relação à média. Já a categoria $\mathrm{MB}$ foi a que apresentou os menores valores de CV, entre 3,41\% e 4,26\%, indicando que essa classe foi a que apresentou a menor variabilidade dentro das classes, com os valores dos pixels muito próximos entre si.

Os resultados acima citados demonstram grande homogeneidade no padrão de cobertura do solo dentro de cada categoria percentual de vegetação, porém apresentam significativa distinção entre elas ao longo do período estudado, fato que pode ser explicado pelo abandono de áreas antes utilizadas pelo homem e que se regeneram gradualmente.

Situação similar foi observada por Muller \& Bessa (2008) ao avaliar a variação do uso do solo na área da APA Guaraqueçaba, estado do Paraná.

A maior presença de pixels das categorias $\mathrm{MB}, \mathrm{MODB}$ e MODA explica-se pela forte presença de vegetação secundária, como capoeira, que varia de mato ralo até um estágio florestal de porte baixo e está intimamente ligada a atividades de pousio e agricultura itinerante. Situação semelhante é observada em estudo realizado por Watrin et al. (2009), que avaliaram a dinâmica temporal da paisagem em antigas áreas de colonização de base econômica familiar, no Pará.

Tabela 1a. Valores de média, desvio-padrão e coeficiente de variação para cada categoria.

Table 1a. Mean, standard deviation and coefficient of variation for each category.

\begin{tabular}{|c|c|c|c|c|c|c|c|c|c|c|c|c|}
\hline \multirow{3}{*}{ Anos } & \multicolumn{12}{|c|}{ Categorias } \\
\hline & \multicolumn{4}{|c|}{ EB } & \multicolumn{4}{|c|}{ MB } & \multicolumn{4}{|c|}{ MODB } \\
\hline & 00 & 03 & 06 & 10 & $\mathbf{0 0}$ & 03 & 06 & 10 & 00 & 03 & 06 & 10 \\
\hline No Pix. & 0 & 10 & 0 & 0 & 346 & 936 & 1567 & 754 & 911 & 950 & 909 & 1343 \\
\hline Med. & & 4 & & & 13 & 11 & 12 & 14 & 25 & 25 & 23 & 22 \\
\hline$\sigma$ & & 0 & & & 2 & 3 & 2 & 2 & 5 & 5 & 5 & 4 \\
\hline CV (\%) & & 0 & & & 19 & 28 & 21 & 11 & 19 & 21 & 20 & 20 \\
\hline
\end{tabular}

No Pix. = Número de pixels; Med. $=$ Média; $\sigma=$ Desvio-padrão; CV $(\%)=$ Coeficiente de variação.

Tabela 1b. Valores de média, desvio-padrão e coeficiente de variação para cada categoria.

Table 1b. Mean, standard deviation and coefficient of variation for each category.

\begin{tabular}{|c|c|c|c|c|c|c|c|c|c|c|c|c|}
\hline \multirow{3}{*}{ Anos } & \multicolumn{12}{|c|}{ Categorias } \\
\hline & \multicolumn{4}{|c|}{ MODA } & \multicolumn{4}{|c|}{ MA } & \multicolumn{4}{|c|}{ EA } \\
\hline & $\mathbf{0}$ & 3 & 6 & 10 & 0 & 3 & 6 & 10 & $\mathbf{0}$ & 3 & 6 & 10 \\
\hline No Pix. & 806 & 640 & 298 & 567 & 594 & 226 & 63 & 173 & 187 & 82 & 7 & 7 \\
\hline Med. & 46 & 42 & 44 & 42 & 60 & 61 & 60 & 60 & 76 & 74 & 74 & 74 \\
\hline$\sigma$ & 6 & 5 & 5 & 6 & 4 & 5 & 4 & 4 & 3 & 3 & 3 & 3 \\
\hline CV (\%) & 13 & 13 & 12 & 13 & 7 & 7 & 6 & 7 & 4 & 4 & 3 & 4 \\
\hline
\end{tabular}

No Pix. = Número de pixels; Med. = Média; $\sigma=$ Desvio-padrão; CV $(\%)=$ Coeficiente de variação. 
A Tabela 2, a seguir, mostra o resultado do teste de Kolmogorov-Smirnov, evidenciando as diferenças de densidade da cobertura vegetal entre as categorias, em cada ano. Nela, os numeros 1, 2, 3, 4, 5, e 6 representam, respectivamente, as categorias $\mathrm{EA}, \mathrm{MA}, \mathrm{MODB}$, MODA, MA e EA.

Esses resultados representam o incremento em termos percentuais, ao longo dos anos, demonstrando o quanto uma categoria difere da outra e o nível percentual de regeneração em que se encontram.

A Figura 4 mostra as diferentes curvas obtidas para quatro anos específicos, separados por intervalos de três anos, mostrando que em 2006 ocorreu uma maior frequência de pixels da classe $\mathrm{MB}$, diferentemente de 2010, quando houve maior frequência de pixels na classe MODB, indicando um aumento da densidade da vegetação. É importante observar que no ano 2000 há uma maior frequência de pixels das classes MA, MODA e EA do que nos demais anos, levando a concluir que houve redução da densidade da vegetação ao longo desses anos.

Além disso, esse fato pode ser explicado por os valores anuais obtidos serem resultantes de várias amostragens, realizadas por várias passagens do satélite ao longo do ano, e por muitas dessas passagens terem ocorrido em datas anteriores ao desmatamento registrado pelo PRODES. Sendo assim, ao se analisar somente as curvas de 2003, 2006 e 2010 pode-se observar que ocorreu redução nas categorias MODB, MODA e MA em relação a 2006, evidenciando a ocorrência de perturbações antrópicas na área e um aumento do número de pixels em MODB e MODA, em 2006 e 2010, respectivamente, indicando uma redução da densidade da vegetação de 2003 a 2006 e um visível aumento de 2006 a 2010.

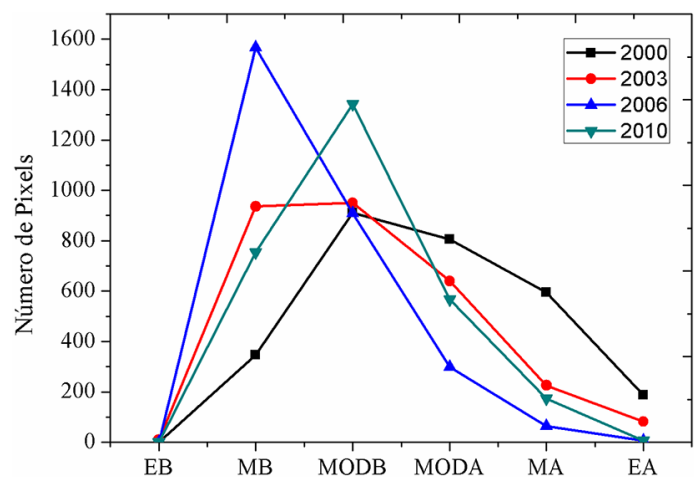

Figura 4. Comportamento da frequência dos pixels dentro das classes nos diferentes anos analisados.

Figure 4. Frequency behavior of the pixels within the classes in different years analyzed.

Tabela 2. Resultados do teste de Kolmogorov-Smirnov.

Table 2. Results of the Kolmogorov-Smirnov test.

\begin{tabular}{|c|c|c|c|c|c|c|c|c|c|c|c|c|c|c|}
\hline 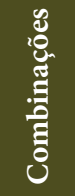 & శ్రి & ్ㅜㅇ & ช్ ชิ & ชి & ర্ণ & 요 & శి & క్తి & ஜ̊ & శి & 응 & $\begin{array}{l}\overline{8} \\
\mathscr{2} \\
\mathscr{N}\end{array}$ & స్ & 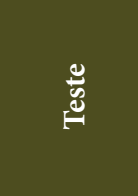 \\
\hline 1 e 2 & 0,12 & 0,30 & 0,24 & 0,33 & 0,31 & 0,41 & 0,55 & 0,40 & 0,51 & 0,14 & 0,27 & 0,55 & 0,03 & Diferentes \\
\hline 1 e 3 & 0,32 & 0,34 & 0,27 & 0,33 & 0,40 & 0,40 & 0,32 & 0,41 & 0,34 & 0,65 & 0,47 & 0,65 & 0,03 & Diferentes \\
\hline $1 \mathrm{e} 4$ & 0,28 & 0,29 & 0,27 & 0,22 & 0,19 & 0,15 & 0,10 & 0,15 & 0,13 & 0,15 & 0,20 & 0,29 & 0,03 & Diferentes \\
\hline 1 e 5 & 0,21 & 0,04 & 0,14 & 0,08 & 0,07 & 0,04 & 0,02 & 0,01 & 0,01 & 0,05 & 0,06 & 0,21 & 0,03 & Diferentes \\
\hline 1 e 6 & 0,07 & 0,03 & 0,08 & 0,03 & 0,02 & 0,00 & 0,00 & 0,00 & 0,00 & 0,00 & 0,00 & 0,08 & 0,03 & Diferentes \\
\hline 2 e 3 & 0,20 & 0,04 & 0,03 & 0,00 & 0,09 & 0,01 & 0,23 & 0,01 & 0,17 & 0,51 & 0,21 & 0,51 & 0,03 & Diferentes \\
\hline 2 e 4 & 0,16 & 0,01 & 0,03 & 0,10 & 0,12 & 0,25 & 0,45 & 0,25 & 0,38 & 0,01 & 0,07 & 0,45 & 0,03 & Diferentes \\
\hline 2 e 5 & 0,09 & 0,25 & 0,10 & 0,25 & 0,24 & 0,37 & 0,53 & 0,39 & 0,50 & 0,09 & 0,20 & 0,53 & 0,03 & Diferentes \\
\hline 2 e 6 & 0,06 & 0,27 & 0,16 & 0,30 & 0,29 & 0,41 & 0,55 & 0,40 & 0,51 & 0,14 & 0,26 & 0,55 & 0,03 & Diferentes \\
\hline 3 e 4 & 0,04 & 0,05 & 0,01 & 0,11 & 0,21 & 0,24 & 0,21 & 0,26 & 0,21 & 0,50 & 0,27 & 0,50 & 0,03 & Diferentes \\
\hline 3 e 5 & 0,11 & 0,30 & 0,13 & 0,25 & 0,33 & 0,36 & 0,30 & 0,40 & 0,33 & 0,60 & 0,41 & 0,60 & 0,03 & Diferentes \\
\hline 3 e 6 & 0,25 & 0,31 & 0,19 & 0,31 & 0,38 & 0,40 & 0,32 & 0,41 & 0,34 & 0,65 & 0,47 & 0,65 & 0,03 & Diferentes \\
\hline 4 e 5 & 0,07 & 0,24 & 0,12 & 0,15 & 0,12 & 0,12 & 0,08 & 0,14 & 0,12 & 0,10 & 0,14 & 0,24 & 0,03 & Diferentes \\
\hline 4 e 6 & 0,22 & 0,26 & 0,19 & 0,20 & 0,17 & 0,15 & 0,10 & 0,15 & 0,13 & 0,15 & 0,20 & 0,26 & 0,03 & Diferentes \\
\hline 5 e 6 & 0,14 & 0,02 & 0,06 & 0,05 & 0,05 & 0,03 & 0,02 & 0,01 & 0,01 & 0,05 & 0,06 & 0,14 & 0,03 & Diferentes \\
\hline
\end{tabular}

$\mathrm{KS} \mathrm{cal}=$ Kolmogorov-Smirnov calculado; KS tab $=$ Kolmogorov-Smirnov tabelado. 
Em um trabalho recente envolvendo pecuária e desmatamento na região Amazônica, os autores Rivero et al. (2009), ao correlacionarem os dados do desmatamento com a pecuária para diferentes cenários, nos anos 2000, 2003 e 2006, evidenciaram o crescimento relativo desses valores $(r=0,6808$, $r=0,7060$ e $r=0,7768$ ) com o passar do tempo. Tais cenários só reforçaram a hipótese já discutida de que a pecuária bovina é a principal causa imediata do desmatamento e que tal tendência tenderá a aumentar (Rivero et al., 2009).

Através desta análise do MDE não é possível inferir sobre a regeneração/recuperação das áreas e sua relação com o relevo, tendo em vista que a maioria das áreas desmatadas são de baixa altitudade e declividade, devido à facilidade de se trabalhar com agricultura e pecuária nessas áreas. Portanto, a maior parte dos pontos referentes a regeneração ocorre nesses sítios (Figura 5) e, nesse caso, não é possível analisar áreas mais declivosas e com altitudes mais elevadas.

De acordo com Weckmüller et al. (2013), os desmatamentos não apresentam altitude ou orientação da encosta preferenciais para sua ocorrência, apenas na declividade é que observa-se uma atuação mais restrita desse fenômeno. Em Plácido de Castro, Acre, não foi encontrado também relação com altitude no estudo da evolução espacial e temporal do desmatamento.

Os resultados obtidos a partir das correlações entre as classes do MOD44B e das imagens do TRMM seguem na Figura 6. Essa figura mostra que o período de 2005 a 2010 apresenta um volume de precipitação superior ao período de 2000 a 2005. Dessa forma, o período de maior precipitação coincide com o período em que os pixels de menor densidade migram para as classes de maior densidade, sugerindo uma possível relação entre os índices de precipitação e o crescimento da vegetação.

O clima na região Amazônica tem sido afetado por condições extremas, como a seca de 2005 (Marengo et al., 2010; Zhang et al., 2008; Cox et al., 2008) e as inundações de 2009 (Marengo, 2010). Todos esses períodos climáticos extremos na região amazônica ocidental são fatores limitantes para o crescimento de florestas em áreas desmatadas.

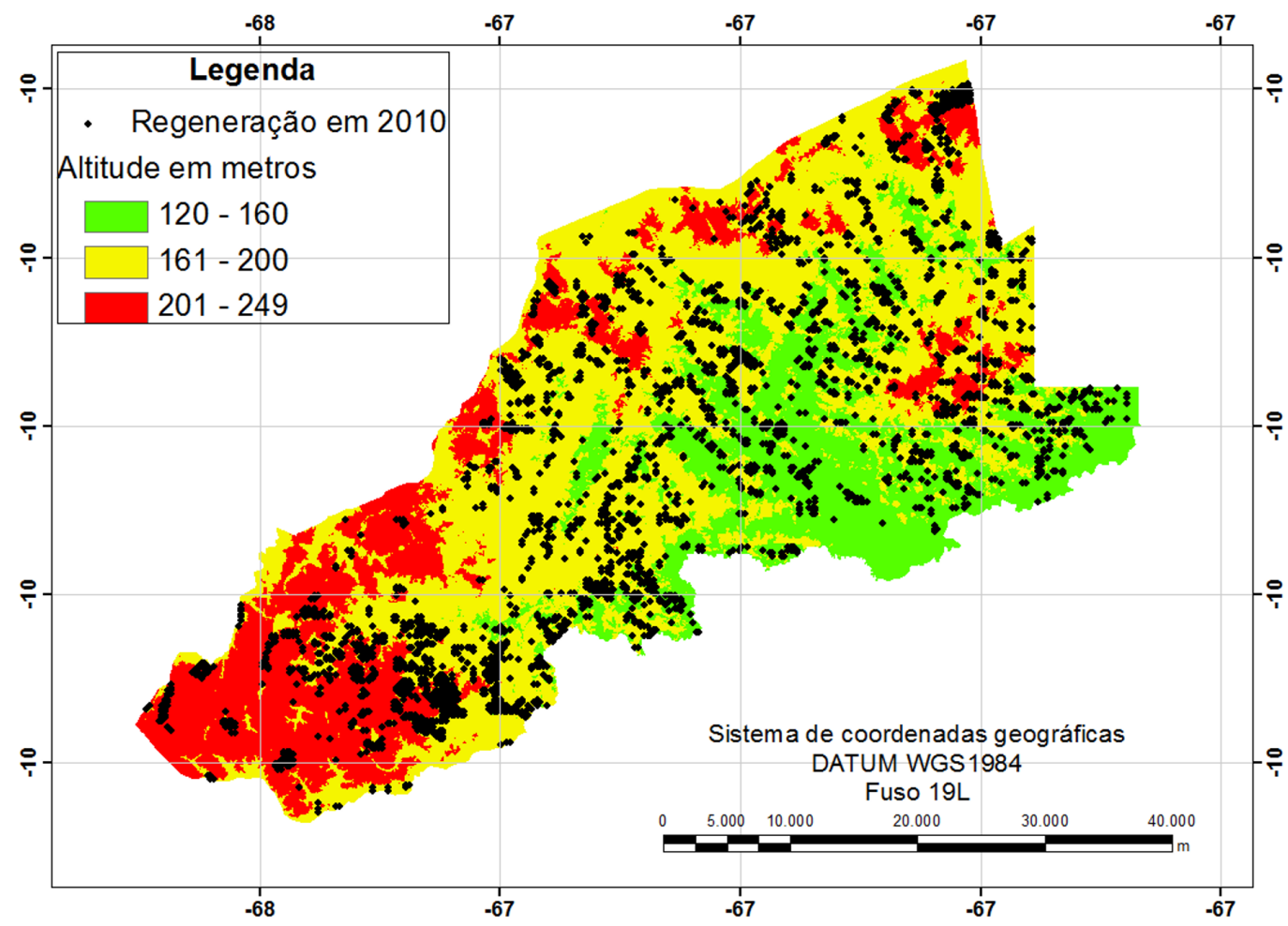

Figura 5. Distribuição dos pontos de regeneração sobre o Modelo Digital de Elevação do SRTM. Figure 5. Distribution of regeneration points on the Digital Elevation Model of the SRTM. 


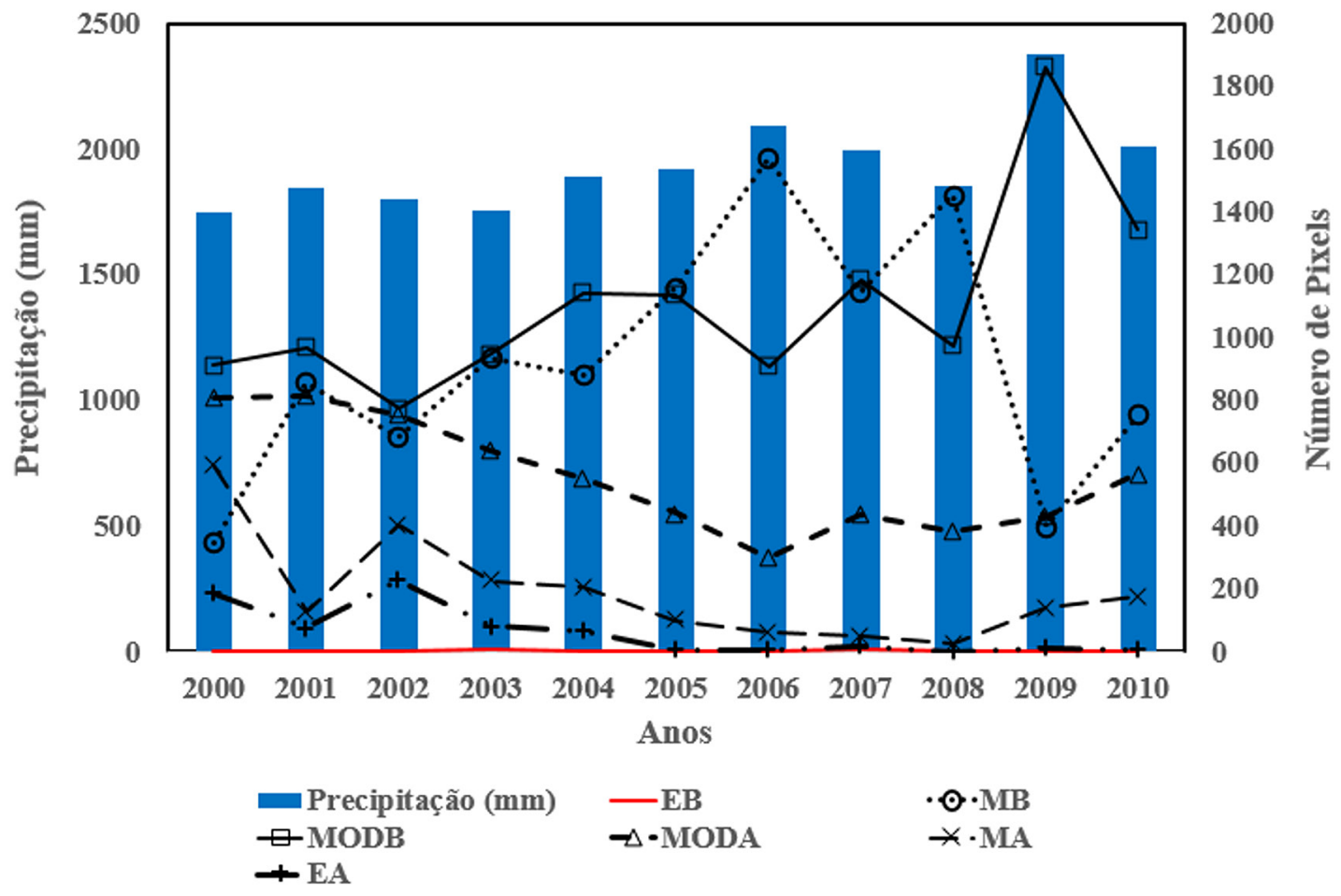

Figura 6. Distribuição das médias anuais de precipitação oriundas do sensor 3B43 e as classes espectrais do MOD44B para o município de Plácido de Castro, AC.

Figure 6. Distribution of mean annual precipitation derived from the sensor 3B43 and spectral classes MOD44B for the city of Plácido de Castro, AC.

Essa relação entre a vegetação e a precipitação indica que a área de estudo pode estar se tornando mais produtiva em termos de biomassa, pois está aumentando sua produção primária bruta, permitindo uma maior disponibilidade hídrica para a recuperação do ecossistema local. Situação semelhante foi descrita no estudo de Zhang et al. (2009), no qual observaram que a relação entre produção primária líquida e produção primária bruta diminuía à medida que se partia de áreas mais secas para áreas com maiores índices de precipitação. Quando essa relação diminui, há o aumento da produção primária bruta, mas há também um elevado consumo de energia pelos organismos presentes no local.

\section{CONCLUSÕES}

O produto MOD44B permitiu verificar que entre 2000 e 2010 ocorreu variação em cada uma das classes de pixels de densidade da cobertura vegetal, dentro de áreas desmatadas quantificadas pelo PRODES, demonstrando a eficácia do produto para a região estudada.

A partir de 2006, as classes de pixel com maior densidade de cobertura florestal aumentaram seu número de pixels, enquanto classes com valores inferiores, como EB e MB, reduziram, levando a concluir que, baseado no MOD44B, está ocorrendo recuperação da vegetação das áreas desmatadas mapeadas pelo PRODES no ano 2000.

Entretanto, não foi possível confirmar a relação do restabelecimento da vegetação com as variações do relevo, pois o histórico de desmatamento da região indica que esse ocorre, na grande maioria das vezes, em áreas mais planas e com menor altitude.

Embora a precipitação aumente no período em que a vegetação aumentou sua densidade por área, estudos futuros são necessários para afirmações concretas acerca da relação da regeneração com o comportamento da precipitação. 


\section{AGRADECIMENTOS}

Os autores agradecem ao CNPq e à CAPES pelo auxílio financeiro, processo 477207/2011-1, e pela bolsa de mestrado do primeiro autor.

\section{STATUS DA SUBMISSÃO}

Recebido: 23 mar., 2014

Aceito: 13 nov., 2015

\section{AUTOR(ES) PARA CORRESPONDÊNCIA}

\section{Rafael Coll Delgado}

Departamento de Ciências Ambientais, Universidade Federal Rural do Rio de Janeiro UFRRJ, Rodovia BR 465, Km 07, CEP 23890-000, Seropédica, RJ, Brasil e-mail: rafaelcoll@ufrrj.br

\section{APOIO FINANCEIRO}

CAPES n. proc. 477207/2011-1.

\section{REFERENNCIAS}

Alves DS, Morton DC, Batistella M, Roberts DA, Souza C Jr. The changing rates and patterns of deforestation and land use in Brazilian Amazonia. Geophysical Monograph Series 2009; 186: 11-23.

Alves DS, Pereira JLG, Sousa CL, Soares JV, Yamaguchi F. Characterizing landscape changes in central Rondônia using Landsat TM imagery. International Journal of Remote Sensing 1999; 20(14): 2877-2882. http://dx.doi. org/10.1080/014311699211859.

Alves DS. Space-times dynamics of deforestation in Brazilian Amazon. International Journal of Remote Sensing 2002; 23(14): 2903-2908. http://dx.doi.org/10.1080/01431160110096791.

Aragão LEO, Malhi Y, Roman-Cuesta RM, Saatchi S, Anderson LO, Shimabukuro YE. Spatial patterns and fire response of recent Amazonian droughts. Geophysical Research Letters 2007; 34(7): L07701. http://dx.doi. org/10.1029/2006GL028946.

Araújo EJG, Pelissari AL, David HC, Miranda ROV, Netto SP, Morais VA et al. Relações dendrométricas em fragmentos de povoamentos de pinus, em Minas Gerais. Pesquisa Florestal Brasileira 2012; 32(72): 355-366. http:// dx.doi.org/10.4336/2012.pfb.32.72.355.

Cox PM, Harris PP, Huntingford C, Betts RA, Collins M, Jones $\mathrm{CD}$ et al. Increasing risk of amazonian drought due to decreasing aerosol pollution. Nature 2008; 453(7192): 212-215. http://dx.doi.org/10.1038/nature06960. PMid:18464740.

Duarte AF. Variabilidade e tendência das chuvas em Rio Branco, Acre, Brasil. Revista Brasileira de Meteorologia 2005; 20(1): 37-42.

Fearnside PM. Desafios para midiatização da ciência na Amazônia: o exemplo da hidrelétrica de Belo Monte como fonte de gases de efeito estufa. In: Fausto A No, editor. A midiatização da ciência: cenários, desafios, possibilidades [online]. Campina Grande: Editora da Universidade Estadual da Paraíba; 2012. p. 107-123. [citado em 2014 fev. 20]. Disponível em: http://philip.inpa.gov.br/ publ_livres/2012/A\%20Hidrel\%C3\%A9trica\%20de\%20 Belo\%20Monte\%20como\%20fonte\%20de\%20gases\%20 de\%20efeito\%20estufa.pdf

Freitas DM, Delgado RC, Rodrigues RA, Souza LP. Variabilidade espaço-temporal na mudança da paisagem no município de Acrelândia, AC. Enciclopédia Biosfera 2012; v(8): 935-946.

Huffman GJ, Bolvin DT, Nelkin EJ, Wolf DB, Adler RF, $\mathrm{Gu}$ G, et al. The TRMM multi-satellite precipitation analysis (TMPA): quasi-global, multiyear, combinedsensor precipitation estimates at fine scale. Journal of Hydrometeorology 2007; 8(1): 38-55. http://dx.doi. org/10.1175/JHM560.1.

Instituto Brasileiro de Geografia e Estatística - IBGE. Cidades@ [online]. Plácido de Castro: IBGE; 2013. [citado em 2013 mar. 15]. Disponível em: http://cidades.ibge.gov. br/xtras/perfil.php?codmun=120038.

Instituto do Homem e Meio Ambiente da Amazônia - IMAZON. Desmatamento e degradação florestal no bioma Amazônia (2000 - 2010) [online]. Belém: Imazon; 2013. [citado em 2014 jan. 29]. Disponível em: www. imazon.org.br.

Instituto Nacional de Pesquisas Espaciais - INPE. Observação da Terra [online]. São José dos Campos: INPE; 2011. [citado em 2013 Fev 10.]. Disponível em: www.obt.inpe.br

Machado L. A fronteira agrícola na Amazônia. In: Becker BK, Christofoletti A, Davidoch FR, Geiger RPP, editores. Geografia e meio ambiente no Brasil. São Paulo: Hucitec; 1998. p. 181-217.

Malhi Y, Grace J. Tropical forests and atmospheric carbon dioxide. Trends in Ecology \& Evolution 2000; 15(8): 332 337. http://dx.doi.org/10.1016/S0169-5347(00)01906-6. PMid:10884705.

Marengo JA, Ambrizzi T, Rocha RP, Alves LM, Cuadra SV, Valverde MC et al. Future change of climate in South America in the late twenty-first century: intercomparison of scenarios from three regional climate models. Climate Dynamics 2010; 35(6): 1073-1097. http://dx.doi.org/10.1007/ s00382-009-0721-6. 
Marengo JA. Extreme rainfall and the flood of the century in Amazonia 2009. Bulletin of the American Meteorological Society 2010; 97: 149.

Mcguffie K, Hendersonsellers A, Zhang H, Durbridge TB, Pitman AJ. Global climate sensitivity to tropical deforestation Glob. Planet. Change 1995; 10(1-4): 97-128. http://dx.doi.org/10.1016/0921-8181(94)00022-6.

Miranda EE, coord. Brasil em relevo [online]. Campinas: Embrapa Monitoramento por Satélite; 2005. [citado em 2013 jun. 17]. Disponível em: http://www.relevobr.cnpm. embrapa.br

Muller ACP, Bessa O Jr. Variação temporal e espacial da cobertura vegetal da floresta ombrófila densa na Área de Proteção Ambiental de Guaraqueçaba no Estado do Paraná, Brasil. Desenvolvimento e Meio Ambiente 2008; 17: 111-119.

NASA's Earth Observing System Data and Information System - NASA EOSDIS [online]. 2013. [citado em 2013 fev. 10]. Disponível em: http://reverb.echo.nasa.gov/reverb

Rivero S, Almeida O, Ávila S, Oliveira W. Pecuária e desmatamento: uma análise das principais causas diretas do desmatamento na Amazônia. Nova Economia 2009; 19(1): 41-66. http://dx.doi.org/10.1590/S0103-63512009000100003.

Running SW, Justice CO, Salomonson V, Hall D, Barker J, Kaufmann YJ et al. Terrestrial remote sensing science and algorithms planned for EOS/MODIS. International Journal of Remote Sensing 1994; 15(17): 3587-3620. http:// dx.doi.org/10.1080/01431169408954346.

Scolforo JRS, Thiersch CR. Biometria florestal: modelos de crescimento e produção florestal. Lavras: UFLA;FAEPE; $2006.393 \mathrm{p}$.

Soares-Filho BS, Nepstad DC, Curran L, Cerqueira GC, Garcia RA, Ramos CA et al. Cenários de desmatamento para a Amazônia. Estudos Avançados 2005; 19(54): 137152. http://dx.doi.org/10.1590/S0103-40142005000200008.
Townshend JRG, Carroll MC, Dimiceli R, Sohlberg M, Hansen RF. Vegetation continuous fields MOD44B, 2001 percent tree cover, collection 5 [online]. Maryland: University of Maryland;College Park; 2001. [citado em 10 fev. 2013]. Disponível: http://www.landcover.org/library/ guide/VCF_C5_UserGuide_Dec2011.pdf

Tropical Rainfall Mapping Mission - TRMM. Mission overview [online]. Washington: NASA; 2013. [citado em 2013 jan. 10]. Disponível em: http://trmm.gsfc.nasa.gov.

United States Geological Survey - USGS. U.S. Geological Survey [online]. USGS; 2013. [citado em 2013 fev. 10]. Disponível em: http://www.usgs.gov.

Vilanova RS, Andrade MSS, Delgado RC, Oliveira JF Jr, Santos RDB. Cobertura florestal da Tectona grandis L. f. Baseado nos produtos MOD44B e 3B43 em Plácido de Castro, AC. Revista Enciclopédia Biosfera 2013; 9(17): 681-695.

Watrin OS, Gerhard P, Maciel MNM. Dinâmica do uso da terra e configuração da paisagem em antigas áreas de colonização de base econômica familiar, no nordeste do estado do Pará. Geografia 2009; 34(3): 455-472.

Weckmüller R, Slovincski NC, Vincens RS. Análise multitemporal como subsídio à identificação da trajetória evolutiva do uso e cobertura da terra no corredor ecológico do Muriqui/RJ. Revista Brasileira de Cartografia 2013; 65(3):467-477.

Werth D, Avissar R. The local and global effects of Amazon deforestation. Journal of Geophysical Research: Atmospheres 2002;107(D20): LBA 55-1-LBA 55-8.

Zhang Y, Xu M, Chen H, Adams J. Global pattern of NPP to GPP ratio derived from MODIS data: effects of ecosystem type, geographical location and climate. Global Ecology and Biogeography. Global Ecology and Biogeography 2009; 18(3): 280-290p. http://dx.doi.org/10.1111/j.14668238.2008.00442.x. 